\title{
Computational Atlases of Severity of White Matter Lesions in Elderly Subjects with MRI
}

\author{
Stathis Hadjidemetriou ${ }^{1}$, Peter Lorenzen ${ }^{1}$, Norbert Schuff ${ }^{2}$, Susanne Mueller ${ }^{2}$, \\ and Michael Weiner ${ }^{2}$ \\ ${ }^{1}$ NCIRE/VA UCSF, 4150 Clement St., San Francisco, CA 94121, USA \\ ${ }^{2}$ UCSF, 4150 Clement St., San Francisco, CA 94121, USA
}

\begin{abstract}
MRI of cerebral white matter may show regions of signal abnormalities. These changes may be associated with hypertension, inflammation, or ischemia, as well as altered brain function. The goal of this work has been to construct computational atlases of white matter lesions that represent both their severity as well as the frequency of their occurrence in a population to achieve a better classification of white matter disease. An atlas is computed with a pipeline that uses 4T FLAIR and $4 \mathrm{~T}$ T1-weighted (T1w) brain images of a group of subjects. The processing steps include intensity correction, lesion extraction, intra-subject FLAIR to $\mathrm{T} 1 \mathrm{w}$ rigid registration, and seamless replacement of lesions in T1w images with synthetic white matter texture. Subsequently, the $\mathrm{T} 1 \mathrm{w}$ images and lesion images of different subjects are registered nonrigidly to the same space. The decrease in $\mathrm{T} 1 \mathrm{w}$ intensities is used to obtain severity information. Atlases were constructed for two groups of subjects, elderly normal controls or with mild cognitive impairment, and subjects with cerebrovascular disease. The lesion severities of the two groups have a significant statistical difference with the severity in the atlas of cerebrovascular disease being higher.
\end{abstract}

Keywords: Intensity correction, brain lesion segmentation, lesion severity, computational atlas construction.

\section{Introduction}

In the aging brain, MRI of cerebral white matter can exhibit increased intensity on T2-weighted (T2w) images and decreased intensity on T1-weighted (T1w) images, these effects are attributed to white matter lesions and MRI is the method of choice for their detection. They have been associated with hypertension, inflammation, or ischemia. They can also decrease cognitive performance, and have been associated with a variety of pathological conditions that include Alzheimer's disease [1, multiple sclerosis, and stroke. However, knowledge of their pathological underpinnings is limited [1].

Several protocols exist, mostly based on a T2w MR image, for the qualitative visual rating of age related lesions 22. However, the visual protocols are not all mutually consistent and can saturate. The qualitative evaluations can also be time consuming, biased, and rarely use the radiometric information of the lesions.

D. Metaxas et al. (Eds.): MICCAI 2008, Part I, LNCS 5241, pp. 450-458, 2008.

(C) Springer-Verlag Berlin Heidelberg 2008 
In automatic lesion analysis the use of T2w imaging has been limited due to the overlap between the lesion intensity range and that of the cerebrospinal fluid 3.4. Atlases have been constructed directly from $\mathrm{T} 2 \mathrm{w}$ images in the form of FLAIR with affine co-registration [4] or from binary lesion maps extracted from FLAIR images followed by non-rigid registration [5]. These atlases are being increasingly investigated as means for improved diagnosis. In multiple sclerosis T2w and FLAIR MRI have been found very sensitive to the presence of lesions, but often not very specific to lesion severity [6]. Physical disability in multiple sclerosis has been found to correlate with the T1w intensity of lesions [6].

The segmentation of white matter lesions often involves coregistration of images of multiple contrasts followed by vectorization of their radiometric information [7. Spatial information has also been used with techniques that include normalized cuts 8 and active contours 9 . Lesion segmentation often requires a threshold for their intensities [3] or its determination through manual seeding 8. The latter is time consuming and may be biased. In general, lesion intensities depend on the subject as well as the instrumentation [5] and can vary throughout the image. Lesions can also be diffuse and they can have irregular shapes, which further complicates their extraction 34.

The aim of this work has been to construct computational atlases of white matter lesion information. A FLAIR and a T1w image are co-analyzed, which is expected to provide a better assessment of white matter lesions than either one alone. The intensity values of lesions in FLAIR and T1w images do not correspond uniquely. Thus, the two images are examined serially starting with the FLAIR image whose processing provides the lesion template. The binary classification of the lesions is extended into a gradual one. The atlases represent both the severity of lesions as well as the frequency of their occurrence in a population. The analysis in this study is used to assess lesion severity of elderly subjects with normal or mildly impaired cognition and due to cerebrovascular disease. The two atlases are shown to have a significant statistical difference.

\section{Methods}

The atlases are constructed with a pipeline which uses a $4 T$ FLAIR image $I_{F L A I R}$ and a $4 T \mathrm{~T} 1 \mathrm{w}$ image $I_{T 1 w}$ of $N$ elderly subjects, $i=0, \ldots, N-1$. The FLAIR image is processed first. Its processing starts with a robust correction for the $4 T$ intensity non-uniformities [10]. The correction does not make use of anatomic priors and hence it can accommodate variations in anatomy of elderly subjects such as ventricle enlargement.

\subsection{Segmentation of White Matter Lesions from the FLAIR Image}

The brain region from $I_{F L A I R, i}$ is extracted using BET [11 and is denoised with median filtering. Many focal intensity distortions occur at the peripheral brain region such as those due to magnetic susceptibilities from the nose and ears, blood flow, as well as chemical shift artifacts from extracranial lipids. Hence, 
the brain template is eroded for $6 \mathrm{~mm}$. The self-co-occurrence statistics of the remaining region are analyzed with expectation maximization into a mixture of two Gaussians. The distribution in the low intensity range corresponds to CSF and is backprojected to the image to cancel the ventricle region. The largest connected component of the valid FLAIR region gives the inner region of the parenchyma, $I_{F, P}(\mathbf{x})$, where $\mathbf{x}$ are the voxel coordinates.

The diffuse nature of lesions causes an extensive partial volume between normal and abnormal parenchyma. This results in an overlap between the intensity distribution of the normal appearing parenchyma and that of the lesions, particularly at the intermediate intensity range 34. The intensities corresponding exclusively to normal appearing white matter and abnormal parenchyma are located at the extremes of the dynamic range. The extremes are extracted using reference measures from FLAIR intensity statistics. The first is the intensity of maximum density, which is the mean intensity of the normal appearing white matter, $\mu_{F, P}$. The second is the full width half maximum $(f w h m)$, which gives the standard deviation of the distribution of the normal appearing white matter, $\sigma_{F, P}=2 \sqrt{2 \pi} f w h m$.

In a FLAIR image the lesion intensity is not directly proportional to severity 12. The maximum intensity is reached at the early stages of lesion development and an advanced lesion evolves to a scar which contains a dark water cyst. This makes the maximum lesion intensity a common feature in FLAIR images. It is computed as the maximum image intensity, $I_{F, P, \max }=\max _{\mathbf{x}} I_{F, P}(\mathbf{x})$, in the inner parenchyma. The intensity range considered to correspond exclusively to normal appearing white matter in the inner parenchyma is $\left[0, \mu_{F, P}+\right.$ $\left.0.25\left(I_{F, P, \max }-\mu_{F, P}\right)\right]$. The intensity range considered to correspond exclusively to abnormal white matter is $\left[\left(\mu_{F, P}+0.51\left(I_{F, P, \max }-\mu_{F, P}\right), I_{F, P, \max }\right]\right.$. To avoid false positives in images that do not contain lesions, the intensitity ranges are readjusted considering $z$-scores based on $\mu_{F, P}$ and $\sigma_{F, P}$ of the distribution of the normal appearing white matter. The lower threshold for lesion intensity is set to $\mu_{F, P}+4 \sigma_{F, P}$.

The intensity ranges provide seed regions in a FLAIR image both for the normal appearing and the abnormal parenchyma. These regions initialize the graph cut segmentation algorithm 13, which resolves ambiguous regions with the path of maximum simulated flow to provide the lesion template $L_{i}(\mathbf{x})$. An example of white matter lesion detection is shown in figure 1 (a). The first row shows the FLAIR image of a subject with a high lesion load, the second row shows the extracted white matter lesion template, and in the third row the outer contours of the lesions are superimposed upon the FLAIR image.

\subsection{Assessment of the Severity of White Matter Lesions}

The severity of the lesions is extracted from the T1w image. Its intensity restoration can be confounded by the presence of white matter lesions whose statistics have been shown to have a variance larger than those of the normal appearing white matter [14. Thus, prior to the intensity restoration of a T1w image, $I_{T 1 w, i}$, the intensity corrected FLAIR image is co-registered rigidly to $I_{T 1 w, i}$ with 


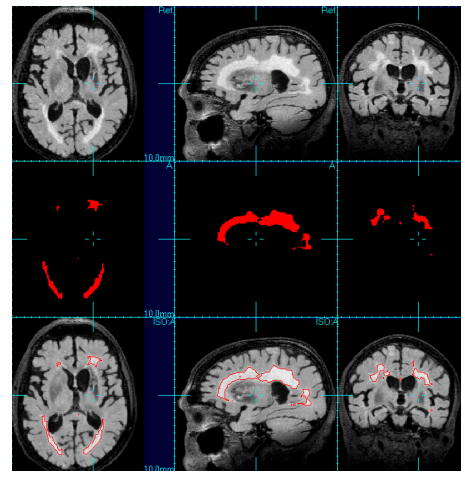

(a)

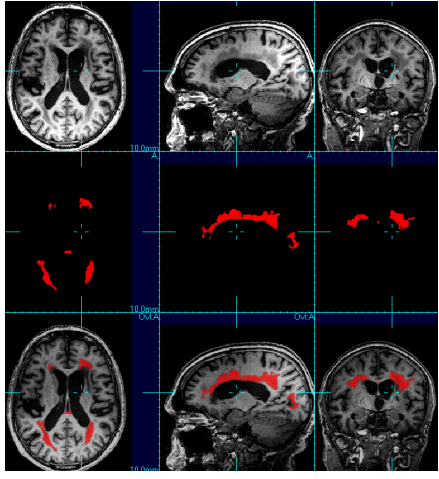

(b)

Fig. 1. Segmentation of white matter lesions from the FLAIR image in (a). The lesions are nulled in the T1 image in (b), prior to its intensity correction.

transformation $f_{i}$. The same transformation is applied to the lesion mask $L_{i}(\mathbf{x})$ to project it to the $T 1 w$-image $I_{T 1 w, i}$ and the regions corresponding to the projection are nulled. An example of the lesion template of a subject in the space of the T1w image is shown in figure 1 (b). The remaining region corresponds to normal appearing parenchyma and is corrected for intensity uniformity [10. The estimated non-uniformity over the healthy region is kept fixed, and is extrapolated to the region occupied by the abnormal tissue numerically with the Laplacian $\nabla B_{T 1 w, i}=0$, where $B_{T 1 w, i}$ is the intensity non-uniformity of the $T 1 w$ image.

The darkening of the white matter in a $\mathrm{T} 1 \mathrm{w}$ image is used to assess lesion severity. The region of the normal parenchyma in the $\mathrm{T} 1 \mathrm{w}$ image is segmented into white matter, gray matter, and cerebrospinal fluid. The segmentation is performed by analyzing the self-co-occurrence statistics into a Gaussian mixture using expectation maximization. The mean intensity of the white matter, $\mu_{w m, i}$, and the mean intensity of the cerebrospinal fluid, $\mu_{c s f, i}$, are used as references for voxel-wise severity evaluation. The mean intensity of the white matter, $\mu_{w m, i}$ corresponds to zero severity. As the lesion intensity in a T1w image, $I_{T 1 w, i}(\mathbf{x})$, decreases towards the mean intensity of the cerebrospinal fluid, in cysts, the severity index increases linearly. The lesion severity index $S_{i}(\mathbf{x})$ at voxel $\mathbf{x}$ is postulated as:

$$
S_{i}(\mathbf{x})=\frac{\left(\mu_{w m, i}-I_{T 1 w, i}(\mathbf{x})\right)}{\left(\mu_{w m, i}-\mu_{c s f, i}\right)} .
$$

Voxels with intensity higher than $\mu_{w m, i}$ are also considered as normal. An example of severity assessment for white matter lesions is shown in figure 2 (a) and in the third row it is superimposed upon the T1w image.

\subsection{Seamless Lesion Replacement in T1w Images}

The intensity corrected T1w images are further processed to replace white matter lesions with synthetic texture representing healthy white matter. To this end 


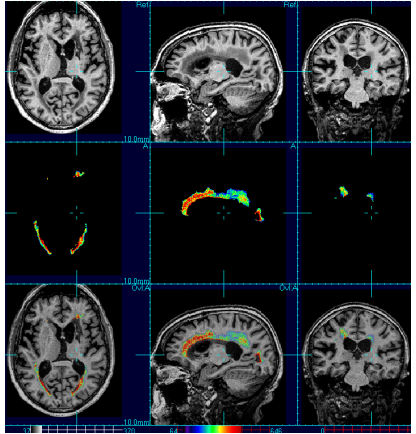

(a)

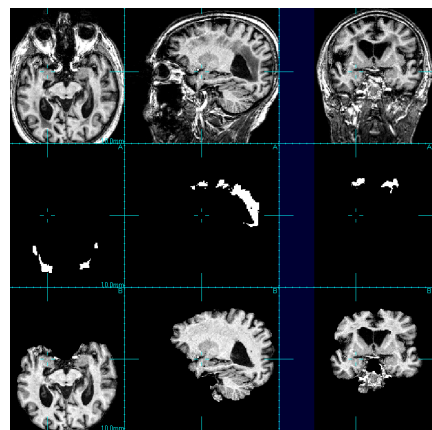

(b)

Fig. 2. An intensity corrected T1 image together with its severity map in (a). Replacement of white matter lesion regions in the third row in (b).

the image is smoothed with median filtering and the brain region is eroded to eliminate bright artifacts in the outer brain. Then, the voxel of maximum intensity is selected, which corresponds to normal appearing white matter. Starting from the selected voxel a region growing algorithm is used to extract a part of the normal appearing white matter. This region is used to provide a sample of the histogram and intensity co-occurrences of the normal appearing white matter.

A texture is randomly generated with approximately the same zero order histogram as that of the normal appearing white matter [1415. The generated texture is altered iteratively to also enforce the local co-occurrence statistics of the normal appearing white matter $C_{N A W M, i}$ into the synthetic white matter $C_{\text {synth,i }}^{t}$, where $t$ is the iteration. The match criterion between the co-occurrences is the $L_{2}$ norm. A pair of neighboring voxels in the synthetic texture is randomly selected. Their intensities are interchanged and the co-occurrence matrix is updated efficiently by considering only the co-occurrences of the two voxels. The interchanges that improve the match of the co-occurrences,

$$
\left\|C_{\text {synth }, i}^{t+1}-C_{N A W M, i}\right\|_{2}<\left\|C_{\text {synth }, i}^{t}-C_{N A W M, i}\right\|_{2},
$$

are retained 14. The iterations continue until the rate of retained changes is very low. A possible discontinuity at the boundary is eliminated by randomly interchanging the intensities of the local cross-border co-occurrences 15. In the first row of figure 2 (b) is an example of an image with a considerable white matter lesion load. In the second row is the extracted lesion image in T1w space, and in the last row the lesions are replaced with synthetic white matter texture.

\subsection{Spatial Normalization to a Common T1w Anatomic Space}

The atlases are created with an unbiased method based on Frechet means in metric spaces 16. It uses an iterative algorithm for the simultaneous deformation of the subject images into an evolving average image. The images are 
transformed with the large deformation fluid based algorithm performed in multiresolution [16. A common T1w atlas space is constructed from the T1w images of several healthy subjects in their sixties. The common space is used as a reference for the coregistration of the lesion replaced $\mathrm{T} 1 \mathrm{w}$ images with 9-parameter affine transformations $h_{a, i}^{-1}$. The co-registered images are further transformed non-rigidly, $h_{n r, i}^{-1}$, to form an unbiased atlas [16]. The concatenation of the intra-subject and inter-subject registrations are applied to every lesion template, $S_{i}^{R}(\mathbf{x})=S_{i}\left(f_{i} h_{a, i}^{-1} h_{n r, i}^{-1} \mathbf{x}\right)$, to transform them to the common T1w space. The co-registered severity maps in a group are averaged to give the atlas:

$$
S^{G}(\mathbf{x})=\frac{1}{N} \sum_{i=0}^{N-1} S_{i}^{R}(\mathbf{x}),
$$

which represents lesions severity as well as population frequency.

\section{Experimental Results}

Two groups of subjects were drawn each from a different study. A group of 15 subjects that were normal or with mild cognitive impairment, but not demented (mean age of $72+/$ - st.dev. of 9,10 men/5 women). The second was a group of 7 subjects diagnosed with cerebrovascular disease with varying degrees of cognitive impairment (mean age of $66+/$ - st.dev. of 8,2 men/5 women). All subjects or their legal guardian provided written informed consent before participating in the respective studies. The protocol was approved by the Committees of Human Research at UCSF and VA. The brain images of the subjects were acquired with a 4Tesla whole-body Siemens/Bruker MRI scanner. The T1w images $I_{T 1 w, i}$ were volumetric based on MPRAGE acquisitions. Their nominal resolution was $1.0 \times 1.0 \times 1.0 \mathrm{~mm}^{3}$ at a matrix of $176 \times 256 \times 256$ voxels. The FLAIR acquisitions

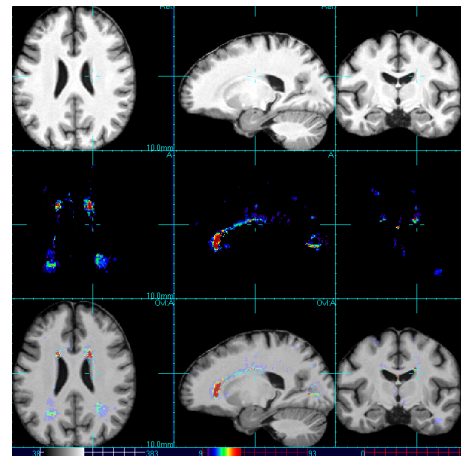

(a)

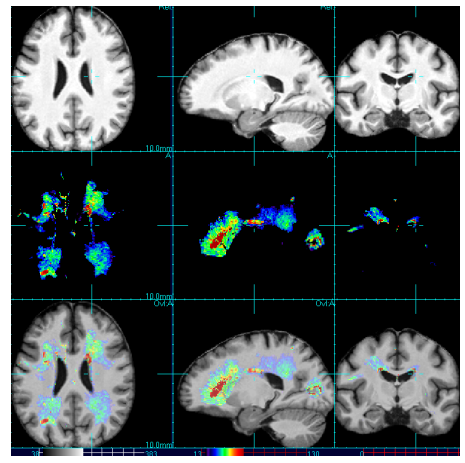

(b)

Fig. 3. The lesion severity atlas of the elderly subjects in (a) and of the subjects with cerebrovascular disease in (b) in the common anatomic space 


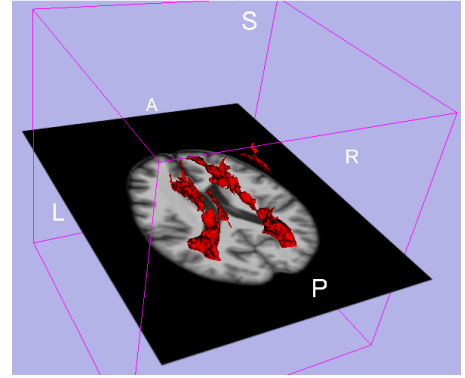

(a)

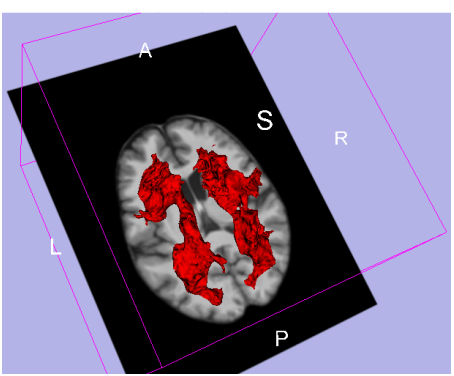

(b)

Fig. 4. Visualizations of the severity atlases shown in figure 3 (a) and figure 3 (b), in (a) and (b), respectively, at the 0.8 iso-severity-frequency level

$I_{F L A I R, i}$ were also volumetric. Their nominal resolution was $1.0 \times 1.0 \times 1.0 \mathrm{~mm}^{3}$ at a matrix size of $176 \times 224 \times 256$ voxels.

The atlas of the elderly subjects that were normal or with mild cognitive impairment is shown with cross-sections in figure 3 (a). The first row shows the reference T1w atlas. The second row shows the combined atlas of lesion severity and frequency in the population. In the third row is the combined lesion atlas superimposed over the reference T1w atlas. The atlas in figure 3(a) is also shown with a simulation of a viewpoint in $3 \mathrm{D}$ at the 0.8 iso-severity-frequency contour in figure 4 (a). Similarly, figure 3 (b) shows cross-sections of the atlas of the subjects with cerebrovascular disease and in figure 4 (b) is a viewpoint of the atlas in $3 \mathrm{D}$ at the 0.8 iso-severity-frequency contour. The lesion severity of the elderly subjects diagnosed with cerebrovascular disease is significantly higher. The two atlases have been compared with a voxelwise Wilcoxon signed rank test. They have a significant statistical difference with $p \approx 0$.

\section{Discussion}

A lesion severity atlas is computed, which uses the FLAIR images to localize lesions and the radiometric information of the $\mathrm{T} 1 \mathrm{w}$ images to assess their severity. The method used provides an unbiased atlas that represents both lesion severity as well as frequency in a population. The atlases computed in this work show that lesion severity of elderly subjects with cerebrovascular disease is significantly higher than that of elderly subjects that were normal or with mild cognitive impairment. The non-rigid registration of images with enlarged ventricles or small brains to a reference atlas of normal subjects can increase the volume of the lesions in the atlas. An atlas can be further processed to classify the lesion templates into categories such as periventricular versus deep white matter.

The severity of the lesions is assessed from the $\mathrm{T} 1 \mathrm{w}$ intensity because it varies monotonically with lesion development, in contrast to the T2-weighted intensity 
of FLAIR, which is designed to suppresses the signal from the cerebrospinal fluid that advanced lesions may contain [12]. Hence, the severity of advanced lesions may be underestimated if the FLAIR image intensities are used alone. Moreover, the $\mathrm{T} 1 \mathrm{w}$ intensity has been found more specific than the $\mathrm{T} 2 \mathrm{w}$ information in terms of lesion severity, primarily in multiple sclerosis [6]. The severity scale can be improved by considering the non-linearity between the intensities in a T1MPRAGE image and relaxation as well as by using an inter-subject reference for the healthy white matter intensity.

\section{References}

1. Erkinjuntti, T., Gao, F., Lee, D.: Lack of difference in brain hyperintensities between patients with early Alzheimer's disease and control subjects. Arch. Neurol. 51, 260-268 (1994)

2. Pantoni, L., Simoni, M., Pracucci, G.: European task on age-related white matter changes. Visual rating scales for age-related white matter changes (leukoaraiosis). Can the heterogeneity be reduced? Stroke 33, 2827-2833 (2002)

3. Jack, C., O’Brien, P., Rettman, D., Shiung, M., Xu, Y., Muthupillai, R., Manduca, A., Avula, R., Erickson, B.: FLAIR histogram segmentation for measurement of leukoaraiosis volume. Journal of Magnetic Resonance Imaging 14, 668-676 (2001)

4. Wen, W., Sachdev, P.: The topography of white matter hyperintensities on brain MRI in healthy 60-to 64-year-old individuals. Neuroimage 22, 144-154 (2004)

5. Yoshita, M., Fletcher, E., DeCarli, C.: Current concepts of analysis of cerebral white matter hyperintensities on magnetic resonance imaging. Topics in Magnetic Resonance Imaging 16(6), 399-407 (2005)

6. Walderveen, M., Kamphorst, W., Scheltens, P.: Histopathologic correlate of hypointense lesions on T1-weighted spin-echo MRI in multiple sclerosis. Neurology 50, 1282-1288 (1998)

7. Pham, S., Shen, D., Herskovits, E., Resnick, S., Davatzikos, C.: Automatic segmentation of white matter lesions in T1-weighted brain MR images. In: Proc. of ISBI, pp. 253-256 (2002)

8. Ballin, A., Galun, M., Gomori, M., Filippi, M., Valsasina, P., Basri, R., Brandt, A.: An integrated segmentation and classification approach applied to multiple sclerosis analysis. In: Proc. of CVPR, pp. 1122-1129 (2006)

9. Warfield, S., Dengler, J., Zaers, J., Guttmann, C., Wells, W., Ettinger, G., Hiller, J., Kikinis, R.: Automatic identification of grey matter structures from MRI to improve the segmentation of white matter lesions. Journal of Image Guided Surgery 1(6), 326-338 (1995)

10. Hadjidemetriou, S., Studholme, C., Mueller, S., Weiner, M., Schuff, N.: Restoration of MRI data for field nonuniformities using high order neighborhood statistics. In: Proc. of SPIE Medical Image Processing, vol. 6512 (2007)

11. Smith, S.: Fast robust automated brain extraction. Proc. of Human Brain Mapping 17, 143-155 (2002)

12. Rovaris, M., Comi, G., Rocca, M., Cercignani, M., Colombo, B., Santuccio, G., Filippi, M.: Relevance of hypointense lesions on fast fluid-attenuated inversion recovery $\mathrm{MR}$ images as a marker of disease severity in cases of multiple sclerosis. American Journal of Neuroradiology 20, 813-820 (1999) 
13. Boykov, Y., Veksler, O., Zabih, R.: Fast approximate energy minimization via graph cuts. IEEE Trans. on PAMI 23(11), 1222-1239 (2001)

14. Kovalev, V., Kruggel, F., Gertz, H., Cramon, D.: Three-dimensional texture analysis of MRI brain datasets. IEEE Trans. on Medical Imaging 20(5), 424-433 (2001)

15. Zalesny, A., Ferrari, V., Caenen, G., Gool, L.: Composite texture synthesis. International Journal of Computer Vision 62(1/2), 161-176 (2005)

16. Joshi, S., Davis, B., Jomier, M., Gerig, G.: Unbiased diffeomorphic atlas construction for computational anatomy. Neuroimage 23, S151-S160 (2004) 